\title{
Effect of oral spray with Lactobacillus on growth performance, intestinal development and microflora population of ducklings
}

\author{
Qi Zhang ${ }^{1, a}$, Yuchen Jie ${ }^{1, a}$, Chuli Zhou', Leyun Wang ${ }^{1}$, Liang Huang ${ }^{1}$, Lin Yang ${ }^{1, *}$, and Yongwen Zhu ${ }^{1, *}$
}

\author{
* Corresponding Authors: \\ Lin Yang \\ Tel: +86-13903075253, Fax: +86-020-85285232, \\ E-mail: ylin899@scau.edu.cn \\ Yongwen Zhu \\ Tel: +86-17728102110, Fax: +86-02038882017, \\ E-mail: 408034085@qq.com
}

1 Guangdong Provincial Key Laboratory of Animal Nutrition Control, College of Animal Science, South China Agricultural University, Guangzhou 510642, China

a These authors contributed equally to the present study.

ORCID

Qi Zhang

https://orcid.org/0000-0002-0749-7836

Yuchen Jie

https://orcid.org/0000-0003-0866-1447

Chuli Zhou

https://orcid.org/0000-0001-6222-0496

Leyun Wang

https://orcid.org/0000-0002-7719-6836

Liang Huang

https://orcid.org/0000-0002-0522-2628

Lin Yang

https://orcid.org/0000-0003-0132-9193

Yongwen Zhu

https://orcid.org/0000-0001-8605-4963

Submitted Jan 15, 2019; Revised Mar 22, 2019; Accepted Jun 13, 2019
Objective: The aim of this study is to investigate the effect of oral spray with probiotics on the intestinal development and microflora colonization of hatched ducklings.

Methods: In Exp. 1, an one-way factorial design was used to study the antibacterial activity of the probiotics and metabolites on Escherichia coli (E. coli) without antimicrobial resistance. There were four experimental groups including saline as control and Lactobacillus, Bacillus subtilis, combined Lactobacillus and Bacillus subtilis groups. In Exp. 2, 64-day-old ducklings were allotted to 2 treatments with 4 replicated pens. Birds in the control group were fed a basal diet supplemented with Lactobacillus fermentation in the feed whereas birds in the oral spray group were fed the basal diet and administrated Lactobacillus fermentation by oral spray way during the first week.

Results: In Exp. 1, the antibacterial activities of probiotics and metabolites on E. coli were determined by the diameter of inhibition zone in order: Lactobacillus $>$ combined Lactobacillus and Bacillus subtilis $>$ Bacillus subtilis. Additionally, compared to $E$. coli without resistance, $E$. coli with resistance showed a smaller diameter of inhibition zones. In Exp. 2, compared to control feeding group, oral spray group increased $(\mathrm{p}<0.05)$ the final body weight at $\mathrm{d} 21$ and average daily gain for d 1-21 and the absolute weight of the jejunum, ileum and total intestine tract as well as cecum Lactobacillus amount at $\mathrm{d} 21$.

Conclusion: Lactobacillus exhibited a lower antibacterial activity on E. coli with resistance than $E$. coli without resistance. Oral spray with Lactobacillus fermentation during the first week of could improve the intestinal development, morphological structure, and microbial balance to promote growth performance of ducklings from hatch to $21 \mathrm{~d}$ of age.

Keywords: Ducklings; Intestinal Development; Lactobacillus; Oral Spray

\section{INTRODUCTION}

The immediate post-hatch period is critical for the development of gastrointestinal tract to minimize the mortality and keep uniformity of young poultry [1]. During first post-hatched days the small intestine undergoes dramatic physiological and morphological changes to increase nutrient digestion and absorption [2,3]. For example, the weight and length of small intestine increases more rapidly than the whole body mass and reaches a maximum between 3 and 7 days [4]. In addition, in newly hatched birds a major change in the source of nutrients occurs by switching from yolk nutrition to enteral nutrition [5]. Early nutrient supply to young poultry is essential for improving the intestine growth and nutrient intake [6] via stimulating digestive enzymes secretion and increasing yolk sac nutrient utilization [7]. Therefore, it is necessary to explore whether the use of neonatal probiotics supplements could promote intestinal health early in life to maximize the nutrient efficiency in later life.

Using probiotics to improve the intestine health of poultry is not a new concept, however, a complete understanding of when and how to use probiotics still has great potential. 
The beneficial effects of probiotics served as alternative feed additives to antibiotics on improving the intestinal microbial balance, morphological structure and feed utilization have been proved in poultry production [8]. Therefore, it is important to select probiotics as supplements to aid in the proper development and microflora colonization of the intestinal tract in hatched birds as soon as possible [9]. Due to the dependence on the residual yolk nutrition and the lower feed intake of birds during the first few days post-hatch [10], it is speculated that provision of probiotics supplied by feeding might not be enough to exert some effect on the rapid development of intestine of birds in early life. Therefore, a new method of supplying probiotics using oral spray was adopted to obtain the greater intestinal health and nutrient efficiency in newly hatched birds in the present study. Firstly in vitro, the inhibitory effect of Lactobacillus and metabolites on Escherichia coli (E. coli) (antimicrobial resistance vs non-antimicrobial resistance) was investigated (Exp. 1). Secondly, in vivo, the effect of Lactobacillus fermentation in oral spray way during the first week of on growth performance, intestinal development, and gut flora amount was evaluated in ducklings from hatch to $21 \mathrm{~d}$ of age (Exp. 2).

\section{MATERIALS AND METHODS}

All experimental procedures were approved by the Institutional Animal Care and Use Committee of South China Agricultural University (SCAU-AEC-2010-0416). This study included 2 experiments as follows: the antibacterial activity of probiotics and metabolites on E. coli in vitro (Exp. 1) and the effect of Lactobacillus fermentation in oral spray way during the first week on performance and intestinal development of ducklings from hatch to $21 \mathrm{~d}$ of age in vivo (Exp. 2).

\section{Microorganisms and medium}

Avian E. coli ATCC25922 without antimicrobial resistance was purchased from China General Microbiological Culture Collection Center (Beijing, China), while avian E. coli ATCC 25922 with antimicrobial resistance was kindly provided by Professor Sun in College of Veterinary Medicine of South China Agricultural University (Guangzhou, China). Lactobacillus and Bacillus subtilis were isolated from a commercial product in our lab by conventional microbiological identification methods [11]. E. coli, Lactobacillus and Bacillus subtilis were cultured in mediums (Boyao Biotechnology Company, Shanghai, China) of Eosin-Methyl Blue Agar broth (\#BS1041), MRS broth (\#BS1138), and Nutrient Agar broth (\#BS1002), respectively.

\section{Antimicrobial sensitivity test}

Firstly, Lactobacillus and Bacillus subtilis were fermented in Luria broth (\#BS2078) for $24 \mathrm{~h}$ in anaerobic and aerobic con- ditions to prepare the solutions of probiotics fermentation without filtration, respectively. In Exp. 1, an one-way factorial design was used to study the antibacterial activity of the probiotics and metabolites on E. coli without antimicrobial resistance. There were four experimental groups including saline as control and Lactobacillus, Bacillus subtilis, combined Lactobacillus and Bacillus subtilis groups. The combined mixture was prepared by an equal volume of Lactobacillus and Bacillus subtilis fermentations. Oxford cups were punched into the plates and were loaded with $2 \mathrm{~mL}$ probiotics culture stock solution. After overnight incubation at $37^{\circ} \mathrm{C}$, the diameters of the inhibition zones were determined to examine the antibacterial activity of against test E. coli [12]. All procedures were performed in three replicates for three times. Then, the Lactobacillus fermentation group presenting a greater inhibitory effect on the E. coli was screened and selected to evaluate the antibacterial activity between two $E$. coli sources with and without antimicrobial resistance.

\section{Birds, sample collection and analyses}

In Exp. 2, 64-day-old Cherry Valley ducklings were weighed individually and allotted to 2 treatments with 4 replicated pens of 8 ducklings per pen based on similar body weight (BW). All ducklings were reared on wire floors in an environmentally controlled room with adjustment of temperature and humidity from 0 to $21 \mathrm{~d}$ of age. Birds in the control group were fed a basal diet supplemented with probiotics fermentation in the feed whereas birds in the oral spray group were fed the basal diet and administrated probiotics fermentation by oral spray during the first week. To determine the antibacterial activity of probiotics and metabolites on E. coli in vitro, the screened Lactobacillus was fermented in $50 \mathrm{~g} / \mathrm{L}$ brown sugar solution as prepared like in Exp. 1. For the oral spray group, $1 \mathrm{~mL}$ Lactobacillus fermentation ( $10^{9}$ colony forming units [CFU]/ $\mathrm{mL}$ ) were taken and supplied for each duckling by oral spraying at $1 \mathrm{~d}$ of age. Oral spray produces a spray of balanced tiny droplets of fermentation of Lactobacillus and delivers it directly into duckling's mouth twice each day. The dose of liquid fermented Lactobacillus was increased step-wise by $1 \mathrm{~mL} / \mathrm{d}$ for each duckling until $7 \mathrm{~d}$ of age. For the control group, ducklings were fed the basal diet supplemented with an equal amount of Lactobacillus fermentation to that used in the spray group per pen during the first week. From 8 to $21 \mathrm{~d}$ of age, all ducklings in the two groups were just fed the same basal diet without any Lactobacillus fermentation supplementation or oral spray. Feed intake was recorded each day per pen. The basal diet was formulated to meet or exceed the nutrient requirements recommended by NRC (1994) for ducklings at the starter period. Compositions and nutrient levels of the basal diet are presented in Table 1. Feed and water were provided ad libitum and no mortality of birds were observed throughout the experimental period. At $\mathrm{d} 7$ and 
Table 1. Composition and nutrient levels of the basal diet (as-fed basis)

\begin{tabular}{lc}
\hline Items & \\
\hline Ingredient (\%) & \\
Corn & 56.23 \\
Soybean meal & 25.64 \\
Rapeseed meal & 8.78 \\
Wheat middling & 2.63 \\
Soybean oil & 2.53 \\
Dicalcium phosphate & 1.42 \\
Limestone & 1.09 \\
L-lysine HCl & 0.13 \\
DL-methionine & 0.20 \\
Salt & 0.25 \\
Choline chloride & 0.10 \\
Vitamin and mineral premix ${ }^{1)}$ & 1.00 \\
Total & 100.00 \\
Nutrient levels & Calculated values \\
Metabolizable energy (MJ/kg) & 12.27 \\
Crude protein (\%) & 20.01 \\
Calcium (\%) & 0.92 \\
Total phosphorus (\%) & 0.65 \\
Available phosphorus (\%) & 0.39 \\
Lysine (\%) & 1.10 \\
Methionine (\%) & 0.51 \\
Methionine+cysteine (\%) & 0.81 \\
\hline Provided per kilogam of & \\
\hline
\end{tabular}

1) Provided per kilogram of diet: vitamin $A, 4,000 \mathrm{IU}$; vitamin $D_{3}, 2,000 \mathrm{IU}$; vitamin E, $24 \mathrm{IU}$; thiamine, $2.0 \mathrm{mg}$; riboflavin, $12 \mathrm{mg}$; pyridoxine, $4.0 \mathrm{mg}$; vitamin $\mathrm{B}_{12}, 0.02$ $\mathrm{mg}$; calcium pantothenate, $10 \mathrm{mg}$; folate, $0.15 \mathrm{mg}$; niacin, $50 \mathrm{mg}$; biotin, $0.15 \mathrm{mg}$ Choline (Choline chloride), 1,000 mg; $\mathrm{Cu}\left(\mathrm{CuSO}_{4} \cdot 5 \mathrm{H}_{2} \mathrm{O}\right), 8 \mathrm{mg} ; \mathrm{Fe}\left(\mathrm{FeSO}_{4} \cdot 7 \mathrm{H}_{2} \mathrm{O}\right)$, $80 \mathrm{mg} ; \mathrm{Zn}\left(\mathrm{ZnSO}_{4} \cdot 7 \mathrm{H}_{2} \mathrm{O}\right), 90 \mathrm{mg} ; \mathrm{Mn}\left(\mathrm{MnSO}_{4} \cdot \mathrm{H}_{2} \mathrm{O}\right), 70 \mathrm{mg} ; \mathrm{Se}\left(\mathrm{NaSeO}_{3}\right), 0.3 \mathrm{mg}$; I (KI), $0.4 \mathrm{mg}$.

21, after $12 \mathrm{~h}$ feed withdrawal, birds were weighed by each replicate pen. The average daily gain (ADG), average daily feed intake (ADFI), and feed:gain ratio (F:G) were calculated. At $\mathrm{d} 21$, based on the average BW per pen, 2 birds in each pen were euthanized by $\mathrm{CO}_{2}$ inhalation, and the duodenum, jejunum, and ileum were separated for the measurements of weight and length. The relative weight and length of the duodenum, jejunum, and ileum were calculated based on the BW. Then, segments of about $1.5 \mathrm{~cm}$ from the middle of duodenum, jejunum, and ileum were excised and flushed with ice-cold saline and immediately placed in $4 \%$ paraformaldehyde for morphometric analysis. The indices of villus height, crypt depth and muscular thickness were measured using computer-aided light microscope image analysis as described by Uni et al [4]. The chyme in caecum of one duckling was selected for measuring the content of total colonies, E. coli and Lactobacillus by the plate CFU method [13].

\section{Statistical analyses}

In Exp. 1, the data about the diameter of inhibition zones on E. coli without antimicrobial resistance were analyzed by one-way analysis of variance using the general linear model procedure of SAS 9.2 (SAS, 2009). Differences among means were tested by the least significant difference method. The data about the diameter of inhibition zones between two $E$. coli sources with and without antimicrobial resistance (Exp. 1) and growth performance, intestine weight and length, morphological structure, and cecal flora number of ducklings between the control and oral spray groups were analyzed by an independent samples t-test. Statistical significance was set at $\mathrm{p}<0.05$.

\section{RESULTS}

\section{Diameter of inhibition zone}

In Exp. 1, the diameter of inhibition zones from Lactobacillus group was greater than that from Bacillus subtilis group or combined Lactobacillus and Bacillus subtilis group $(\mathrm{p}<0.01)$, while the diameter of inhibition zones from combined $L a c$ tobacillus and Bacillus subtilis group was greater than that from Bacillus subtilis group ( $<<0.01$, Table 2; Figure 1). Under Lactobacillus fermentation treatment, E. coli with antimicrobial resistance showed a smaller diameter of inhibition zones compared to E. coli without antimicrobial resistance ( $<<0.05$, Table 3; Figure 2).

\section{Growth performance}

In Exp. 2, oral spray with Lactobacillus fermentation during the first week had no effect on the final BW at $21 \mathrm{~d}$ and ADG at $d 1$ to 7 as well as ADFI and F:G at 1 to 7 and $d 1$ to 21 (p> 0.05 ; Table 4). Compared to the control group, oral spray group had an increased final BW at $21 \mathrm{~d}$ and ADG of birds at $\mathrm{d} 1$ to $21(\mathrm{p}<0.05$; Table 4$)$.

\section{Weight and length of intestine}

The data of absolute weight and length of duodenum, jejunum,

Table 2. Effect of probiotics on the diameter of inhibition zone of Escherichia coli without resistance in vitro

\begin{tabular}{|c|c|c|c|c|c|}
\hline \multirow{2}{*}{ Inhibition zone } & \multicolumn{4}{|c|}{ Probiotics groups } & \multirow{2}{*}{ p-value } \\
\hline & Saline & Lactobacillus & Bacillus subtilis & Lactobacillus+Bacillus subtilis & \\
\hline Diameter (mm) & - & $24.4 \pm 0.99^{a}$ & $11.1 \pm 1.16^{c}$ & $19.2 \pm 1.30^{b}$ & $<0.0001$ \\
\hline
\end{tabular}




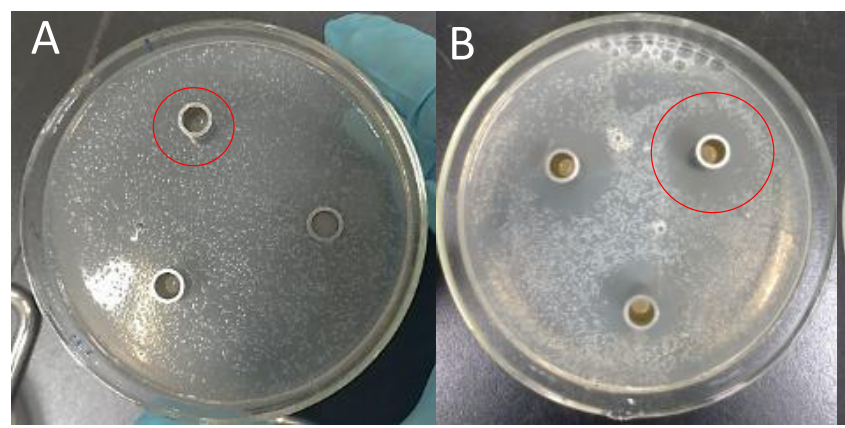

Saline
Lactobacillus

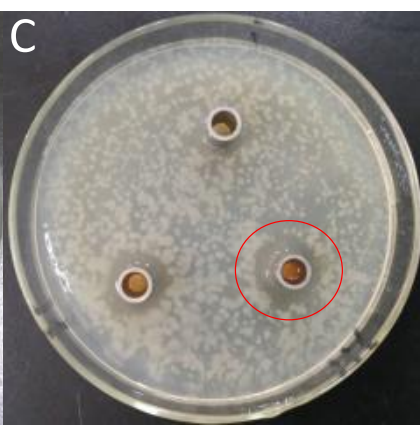

Bacillus subtilis

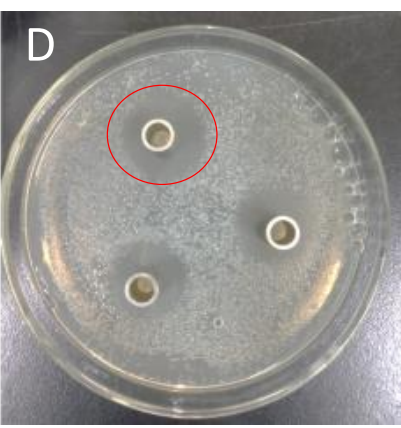

Lactobacillus +

\section{Bacillus subtilis}

Figure 1. Representative inhibition zone of Escherichia coli (E. coli) without resistance. The diameters of the inhibition zones were determined to examine the antibacterial activity of probiotics culture stock solution against test $E$. coli (see red circle). The order of antibacterial activity was that Lactobacillus (B)> combined Lactobacillus and Bacillus subtilis (D) $>$ Bacillus subtilis $(C)>$ saline $(A)$.

Table 3. Effect of Lactobacillus on the diameter of inhibition zone of Escherichia coli with or without resistance in vitro

\begin{tabular}{lcccc}
\hline \multirow{2}{*}{ Inhibition zone } & \multicolumn{3}{c}{ Escherichia coli sources } & Resistance \\
\cline { 2 - 5 } & Saline & Non-resistance & $23.0 \pm 1.23^{\mathrm{b}}$ & \\
\hline Diameter $(\mathrm{mm})$ & - & $24.4 \pm 0.50^{\mathrm{a}}$ & 0.0076 & \\
\hline
\end{tabular}

Data was expressed as mean \pm standard deviation $(n=3)$.

"-" , No detected inhibition zone.

$a, b$ Means within the same row lacking a common superscript differ $(p<0.05)$.

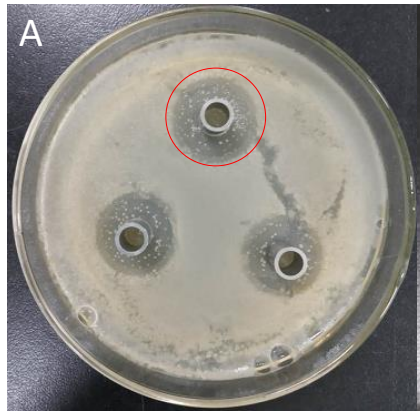

Non-resistance
B

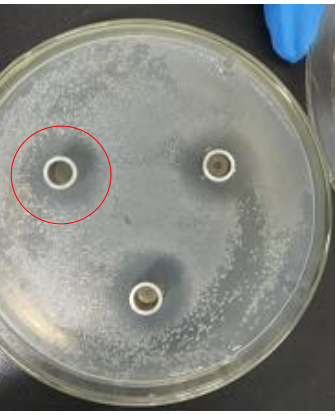

Resistance

Figure 2. Representative inhibition zone of Escherichia coli (E. coli) with or without resistance. The Lactobacillus fermentation with a greater inhibitory effect on the $E$. coli was selected to evaluate the antibacterial activity between two $E$. coli sources with and without antimicrobial resistance (see red circle). The order of antibacterial activity was that non-resistance $(A)>$ resistance $(B)$.

ileum and total intestine tract at $\mathrm{d} 21$ are presented in Table 5. Oral spray group increased $(\mathrm{p}<0.05)$ the absolute weight of jejunum, ileum, and total intestine tract and did not influence other above-mentioned indices $(\mathrm{p}>0.05)$ of ducklings at $\mathrm{d} 21$ compared to the control group.

\section{Intestinal histomorphology}

The data and representative light microscopy of villus height, crypt depth, musculature thicknesses and villus height:crypt depth ratio of duodenum, jejunum and ileum are shown in Table 6, Figure 3, respectively. Oral spray with Lactobacillus fermentation during the first week increased $(\mathrm{p}<0.05)$ the villus height and villus height:crypt depth ratio of duodenum and jejunum and musculature thicknesses of jejunum as well as decreased the crypt depth of duodenum compared

Table 4. Effect of oral spray with Lactobacillus on growth performance of ducklings from hatch to $21 \mathrm{~d}$ of age

\begin{tabular}{llcccc}
\hline Period & Group & Final BW $(\mathbf{g} /$ bird) & ADG (g/d/bird) & ADFI (g/d/bird) & F:G (g/g) \\
\hline D 1-7 & Control & $159.7 \pm 7.7$ & $16.9 \pm 1.4$ & $27.6 \pm 5.3$ & $1.62 \pm 0.20$ \\
& Oral spray & $165.7 \pm 6.0$ & $17.7 \pm 1.1$ & $26.5 \pm 3.6$ & $1.50 \pm 0.18$ \\
& p-value & 0.26 & 0.37 & 0.76 & 0.39 \\
D 1-21 & Control & $740.3 \pm 20.6^{\mathrm{b}}$ & $32.5 \pm 0.9^{\mathrm{b}}$ & $66.4 \pm 4.1$ & $2.05 \pm 0.17$ \\
& Oral spray & $801.1 \pm 37.7^{\mathrm{a}}$ & $35.3 \pm 1.8^{\mathrm{a}}$ & $70.7 \pm 3.8$ & $2.01 \pm 0.15$ \\
& p-value & 0.03 & 0.03 & 0.17 & 0.72 \\
\hline
\end{tabular}

Data was expressed as mean \pm standard deviation $(n=4)$.

$B W$, body weight; $A D G$, average daily gain; $A D F I$, average daily feed intake; $F: G$, feed:gain ratio.

$a, b$ Means within the same column lacking a common superscript differ $(p<0.05)$. 
Table 5. Effect of oral spray with Lactobacillus on the absolute intestinal weight and length of ducklings at $21 \mathrm{~d}$ of age

\begin{tabular}{llcccc}
\hline Items & Group & Duodenum & Jejunum & Ileum & Total tract \\
\hline Absolute weight $(\mathrm{g})$ & Control & $4.45 \pm 0.53$ & $10.0 \pm 0.89^{\mathrm{b}}$ & $9.25 \pm 0.75^{\mathrm{b}}$ & $23.7 \pm 1.7^{\mathrm{b}}$ \\
& Oral spray & $4.84 \pm 0.68$ & $11.3 \pm 1.20^{\mathrm{a}}$ & $10.7 \pm 1.38^{\mathrm{a}}$ & $26.9 \pm 2.7^{\mathrm{a}}$ \\
& p-value & 0.22 & 0.02 & 0.02 & 0.01 \\
Absolute length $(\mathrm{cm})$ & Control & $26.3 \pm 2.0$ & $62.8 \pm 4.6$ & $59.5 \pm 4.4$ & $148.6 \pm 9.4$ \\
& Oral spray & $26.8 \pm 2.0$ & $64.7 \pm 4.0$ & $64.3 \pm 7.2$ & $155.8 \pm 10$ \\
& p-value & 0.63 & 0.39 & 0.13 & 0.16 \\
\hline
\end{tabular}

Data was expressed as mean \pm standard deviation $(n=8)$.

${ }^{a, b}$ Means within the same column lacking a common superscript differ $(p<0.05)$.

Table 6. Effect of oral spray with Lactobacillus on intestinal histomorphology of ducklings at $21 \mathrm{~d}$ of age

\begin{tabular}{llcccc}
\hline Segments & Group & Villus height $(\mu \mathrm{m})$ & Crypt depth $(\mu \mathrm{m})$ & Musculature thicknesses $(\mu \mathrm{m})$ & Villus height:crypt depth \\
\hline Duodenum & Control & $365 \pm 134^{\mathrm{b}}$ & $144 \pm 23^{\mathrm{b}}$ & $542 \pm 136$ & $2.49 \pm 0.64^{\mathrm{b}}$ \\
& Oral spray & $436 \pm 89^{\mathrm{a}}$ & $123 \pm 30^{\mathrm{a}}$ & $585 \pm 74$ & $3.84 \pm 1.43^{\mathrm{a}}$ \\
& p-value & 0.02 & 0.001 & 0.14 & $<0.0001$ \\
Jejunum & Control & $296 \pm 89^{\mathrm{a}}$ & $111 \pm 21$ & $438 \pm 115^{\mathrm{b}}$ & $2.76 \pm 0.99^{\mathrm{b}}$ \\
& Oral spray & $396 \pm 80^{\mathrm{b}}$ & $107 \pm 34$ & $617 \pm 91^{\mathrm{a}}$ & $4.08 \pm 1.68^{\mathrm{a}}$ \\
& p-value & $<0.0001$ & 0.59 & $<0.0001$ & $<0.0001$ \\
Ileum & Control & $325 \pm 45$ & $102 \pm 16$ & $446 \pm 50$ & $3.28 \pm 0.74$ \\
& Oral spray & $343 \pm 67$ & $112 \pm 23$ & $475 \pm 85$ & $3.10 \pm 0.38$ \\
& p-value & 0.24 & 0.07 & 0.11 & 0.24 \\
\hline
\end{tabular}

Data was expressed as mean \pm standard deviation $(n=8)$.

a,b Means within the same column lacking a common superscript differ $(p<0.05)$.

to the control group, but did not affect the intestinal histomorphology of ileum ( $>0.05)$.

\section{Cecal microflora population}

Compared with the control group, oral spray with Lactobacillus fermentation during the first week increased $(\mathrm{p}<0.05$, Table 7) the cecal Lactobacillus amount and had no effect ( $\mathrm{p}>0.05)$ on the amounts of E. coli and total colonies and Lactobacillus: E. coli ratio.

\section{DISCUSSION}

The use of probiotics has become more common to achieve greater productivity and health benefits in the poultry production [8]. Numerous in vivo studies in broilers [14], turkeys [15], and layers [16] have proved that probiotics as feed additive can improve nutrient utilization, gut health, and immune function, resulting in better production performance, such as greater BW gain and resistance to infectious bacteria. However, several other workers reported that no beneficial effects were observed in birds given diets supplemented with or without probiotics $[17,18]$. Variations in the efficacy of probiotics may depend upon the stability and efficiency of the probiotics and species or strains of microorganisms given to the host [19]. Considering the susceptibility to $E$. coli infections in poultry at the post-hatched period, the inhibitory effect of probiotics against $E$. coli was investigated in vitro in the present study. According to the diameter of inhibition zone, the antibacterial activity of probiotics culture was Lactobacillus $>$ combined Lactobacillus and Bacillus subtilis $>$ Bacillus subtilis. As indicated previously [20], the manner by which Lactobacillus inhibits the growth and proliferation of pathogenic bacteria is by lowering the $\mathrm{pH}$ with the production of primary metabolites such as organic acids and hydrogen peroxide. The use of antibiotics in poultry feed as a growth promoter has been restricted in many countries around the world [21]. Probiotics are considered alternative microbial feed additives to enhance growth and disease prevention for birds by improving the intestinal microbial balance [22]. Therefore, in the present study the screened Lactobacillus were selected to evaluate the antibacterial activity between two $E$. coli sources with and without antimicrobial resistance as determined by the diameter of the inhibition zone. Compared to E. coli without resistance, E. coli with resistance displayed a smaller diameter of inhibition zones under Lactobacillus fermentation treatment, implying that $E$. coli with antimicrobial resistance exhibited a greater resistance of Lactobacillus fermentation. It is suggested that the possibility of replacing antibiotics in poultry production might not only depend on one alternative feed additive alone, such as probiotics, enzymes, and acidifiers etc, but requires a comprehensive nutrition strategy together with good breeding and management conditions [20]. 

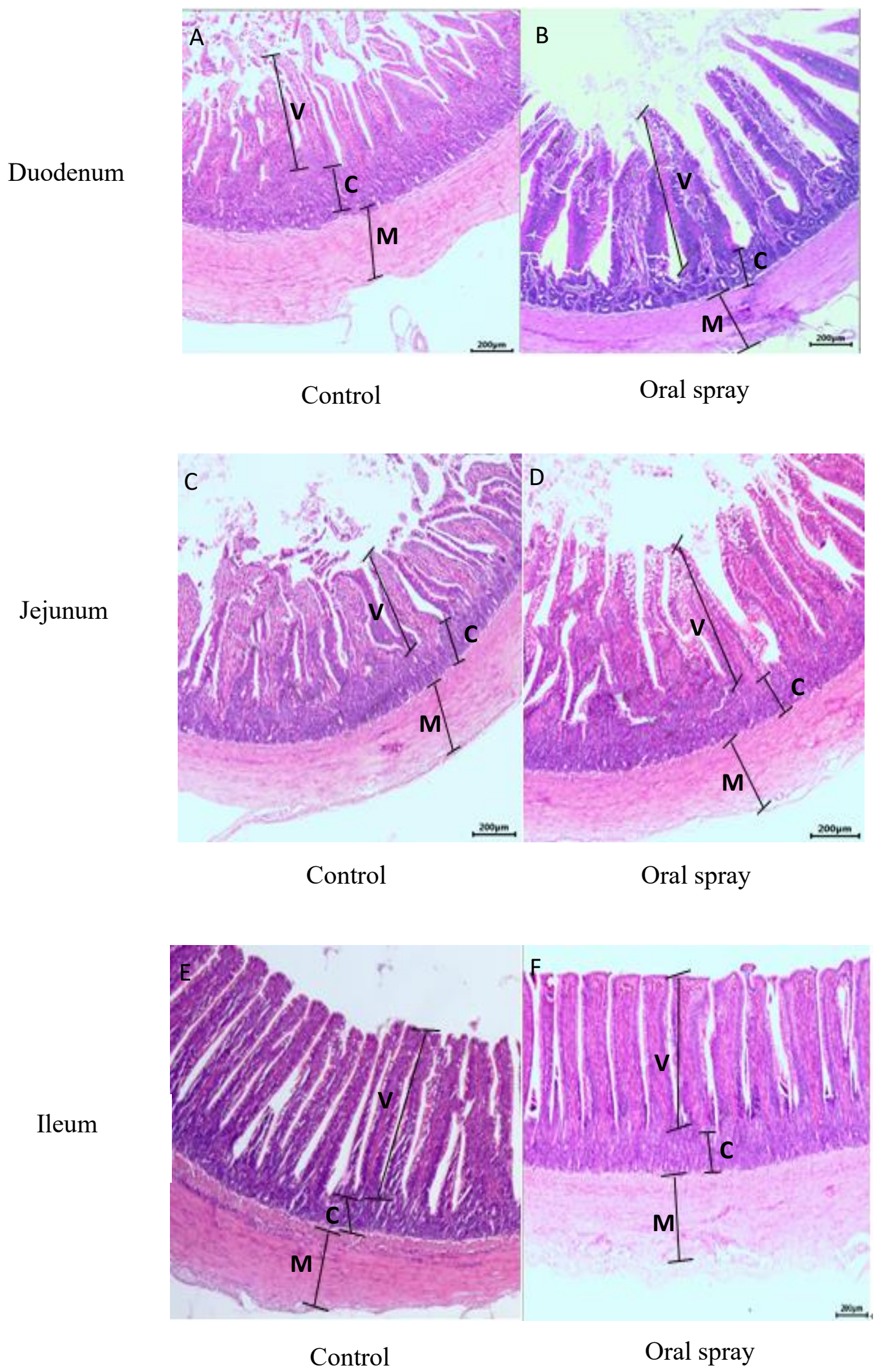

Figure 3. Representative light microscopy $(\times 200)$ of the histomorphology of duodenum $(A, B)$, jejunum $(C, D)$ and ileum $(E, F)$ of the ducklings at $21 \mathrm{~d}$ of age. $V$, villus; C, crypt; M, muscularis mucosa; control group, the basal diet supplemented with Lactobacillus fermentation; oral spray group, ducklings fed the basal diet and administrated Lactobacillus fermentation by oral spray way. 
Table 7. Effect of oral spray with Lactobacillus on cecum flora amount of ducklings at $21 \mathrm{~d}$ of age (log CFU/g)

\begin{tabular}{lcccc}
\hline Items & Escherichia coli & Lactobacillus & Total colonies & Lactobacillus:Escherichia coli ratio \\
\hline Control & $8.65 \pm 0.83$ & $8.30 \pm 0.97^{\mathrm{b}}$ & $7.86 \pm 0.58$ & $0.97 \pm 0.18$ \\
Oral spray & $8.01 \pm 0.46$ & $9.59 \pm 0.15^{\mathrm{a}}$ & $8.46 \pm 0.19$ & $1.20 \pm 0.08$ \\
p-value & 0.22 & 0.038 & 0.09 & 0.06 \\
\hline
\end{tabular}

CFU, colony forming units.

Data was expressed as mean \pm standard deviation $(n=4)$.

${ }^{a, b}$ Means within the same column lacking a common superscript differ $(p<0.05)$.

The key point of adding beneficial bacteria to improve the productive performance and intestine health of poultry lines is in understanding completely when and how to use them. Some studies reported that there are positive effects on production efficiency of broiler chickens fed diets containing Lactobacillus cultures $[14,23,24]$ or Lactobacillus fermentation administered intragastrically [25]. Other reporters found that no significant differences were observed in weight gain of chicken given diets with or without Lactobacillus cultures $[17,18]$. These inconsistent results may be due to the differences in the stability and specificity of the Lactobacillus strain to the host, exact dose and supply way of Lactobacillus as well as the developmental period and nutritional status of the birds. For example, in the newly hatched chick, the small intestinal development and function appears to be immature and should be further improved to minimize the mortality and keep uniformity of young poultry [4]. Therefore, it is important to select probiotics as supplements to aid in the proper development and microflora colonization of the intestinal tract in the early life of hatched birds as soon as possible [9]. In the current study, the beneficial effects of the Lactobacillus fermentation supplements supplied by oral spray were examined. Compared to the feeding control group, oral spray with Lactobacillus fermentation during the first week had no effect on growth performance of ducklings during 1 to $7 \mathrm{~d}$ of age while positively increased the final BW at $21 \mathrm{~d}$ and ADG from 1 to 21 $\mathrm{d}$. Due to the dependence on the residual yolk nutrition and the lower feed intake of birds during the first few days post hatch, it was presumed that provision of probiotics supplied by the traditional feeding way might be insufficient to exert some effect on the rapid development of intestine of birds in early life and then could not maximize the value of the nutrient efficiency compared to oral spraying. In our study, the greater dose of Lactobacillus fermentation by oral spray at the critical post-hatch period might be more conducive to the proper development of the intestinal tract to obtain the greater digestion and utilization of nutrients in long run.

The interaction of intestinal growth, digestive functions, and enteral nutrition are critical for hatched poultry during the post-hatch period [4]. In order to increase digestion and absorption of nutrients from the exogenous feed, physiological and morphological changes of the small intestine are dramatic in birds at the first post-hatched days [2,3]. For example, the weight and length of the small intestine increased more rapidly than the whole body mass and reach a maximum between 3 and 7 days $[3,4]$. Therefore, early nutrient supply to young poultry is essential for improving the intestine growth and nutrient intake [6]. Access to supplements stimulating digestive enzyme and yolk sac nutrient utilization in early life can promote the intestinal development [7]. In the current study, ducklings given Lactobacillus fermentation immediately during the first week exhibited a greater absolute weight of the jejunum, ileum, and total intestine tract. Similar results about promoting intestine development were consistent with those reported in broilers fed probiotics [26]. Therefore, the sooner the gastrointestinal tract achieves functional capacity, the more nutrients can be utilized efficiently, leading to increased weight gain from $\mathrm{d} 1$ to 21 . In addition, the immediate post-hatch period is critical for intestinal morphological development in order to enlarge the intestinal absorptive surface and increase nutrient supply [3]. Thus, some of the enhanced growth effects of early nutrition may be explained by changes in intestinal tract development. Moreover, oral spray with Lactobacillus and metabolites during the first week increased the villus height and villus height:crypt depth ratio of duodenum and jejunum. The improved intestinal morphology was parallel with simultaneously increased intestinal weight by Lactobacillus fermentation administration in the present study, in turn suggesting that oral spray with Lactobacillus fermentation could stimulate intestinal development to improve nutrients digestion and absorption for eventually better growth performance in ducklings at $21 \mathrm{~d}$ of age.

Newly hatched chickens with immature immune function and unstable intestinal flora were susceptible to bacterial infection [9]. Thus, the colonization of beneficial microorganisms should be encouraged to fight against pathogen infection during post-hatch period as soon as possible. An increase amount of Lactobacillus was observed in the cecum of ducklings subjected to oral spray with Lactobacillus fermentation in the present study. Similar results were observed in newborn birds by feeding [24] or inoculation with Lactobacillus strains [27]. Therefore, the improved intestinal microbial environment resulted from Lactobacillus fermentation administration of ducklings in the present study. Since Lactobacillus ad- 
ministration could increase cecal Lactobacillus colonization, Lactobacillus could inhibit cecal harmful bacteria colonization by competition for nutrients and adhesion sites on the intestinal epithelium [28]. However, there is no consistent beneficial effects of Lactobacillus and metabolites on the antibacterial activity of E.coli between Exp. 1 (in vitro) and Exp. 2 (in vivo). In fact, the degree of Lactobacillus effect in vivo depends upon the dose or type/blend of Lactobacillus, the duration of feeding, bird's age, overall hygiene conditions on farm and environmental factors. It is implied that the survival ability and adhesive capability of Lactobacillus as well as the exact dose and duration of Lactobacillus to produce the beneficial effects should be evaluated in our future study. Additionally, E coli colonisations in ducklings reared in a comfortable and clean environment might be kept at a stable and lower level and not be affected easily by Lactobacillus treatment.

In conclusion, Lactobacillus with the better anti-bacteria ability exhibited a lower antibacterial activity on $E$. coli with antimicrobial resistance than $E$. coli without resistance in vitro. In vivo, oral spray with Lactobacillus fermentation during the first week could improve the intestinal development, morphological structure, and microbial balance to promote growth performance of ducklings from hatch to $21 \mathrm{~d}$ of age.

\section{CONFLICT OF INTEREST}

We certify that there is no conflict of interest with any financial organization regarding the material discussed in the manuscript.

\section{ACKNOWLEDGMENTS}

The authors thank Professor Sun lab of Veterinary Medicine of South China Agricultural University for kindly providing avian E. coli with antimicrobial resistance. This paper was sponsored by National Natural Science Foundation of China (31802080), Guangdong Provincial Natural Science Foundation for Starting Ph.D. (2017A030310398 and 2018A030310202), and National Waterfowl Industry Program in China (CARS42-15).

\section{REFERENCES}

1. Uni Z, Ferket RP. Methods for early nutrition and their potential. World's Poult Sci J 2004;60:101-11. https://doi.org/10.1079/ WPS20038

2. Sklan D. Development of the digestive tract of poultry. World's Poult Sci J 2001;57:415-28. https://doi.org/10.1079/WPS200 10030

3. Uni Z, Ganot S, Sklan D. Posthatch development of mucosal function in the broiler small intestine. Poult Sci 1998;77:75-
82. https://doi.org/10.1093/ps/77.1.75

4. Uni Z, Noy Y, Sklan D. Posthatch development of small intestinal function in the poult. Poult Sci 1999;78:215-22. https:// doi.org/10.1093/ps/78.2.215

5. Noy Y, Sklan D. Yolk and exogenous feed utilization in the posthatch chick. Poult Sci 2001;80:1490-5. https://doi.org/ $10.1093 / \mathrm{ps} / 80.10 .1490$

6. Noy Y, Geyra A, Sklan D. The effect of early feeding on growth and small intestinal development in the posthatch poult. Poult Sci 2001;80:912-9. https://doi.org/10.1093/ps/80.7.912

7. Prabakar G, Pavulraj S, Shanmuganathan S, Kirubakaran A, Mohana N. Early nutrition and its importance in poultry: a review. Indian J Anim Nutr 2016;33:245-52. https://doi.org/10. 5958/2231-6744.2016.00044.X

8. Kabir SM. The role of probiotics in the poultry industry. Int J Mol Sci 2009;10:3531-46. https://doi.org/10.3390/ijms10083 531

9. Barnes EM, Impey CS, Cooper DM. Manipulation of the crop and intestinal flora of the newly hatched chick. Clin Nutr 1980;33:2426-33. https://doi.org/10.1093/ajcn/33.11.2426

10. Noy Y, Sklan D. Yolk utilisation in the newly hatched poult. Br Poult Sci 1998;39:446-51. https://doi.org/10.1080/00071669 889042

11. Eisenberg HD. Clinical microbiology procedures handbook. Washington DC, USA: American Society for Microbiology; 1992.

12. Cooper KE. The theory of antibiotic inhibition zones. In: Frederick K, editor. Analytical microbiology. New York, USA: Academic Press; 1963. pp. 1-86.

13. Jett BD, Hatter KL, Huycke MM, Gilmore MS. Simplified agar plate method for quantifying viable bacteria. Biotechniques 1997;23:648-50. https://doi.org/10.2144/97234bm22

14. Huang MK, Choi YJ, Houde R, Lee JW, Lee B, Zhao X. Effects of Lactobacilli and an acidophilic fungus on the production performance and immune responses in broiler chickens. Poult Sci 2004;83:788-95. https://doi.org/10.1093/ps/83.5.788

15. Torres-Rodriguez A, Donoghue AM, Donoghue DJ, Barton JT, Tellez G, Hargis BM. Performance and condemnation rate analysis of commercial turkey flocks treated with a Lactobacillus spp.-based probiotic. Poult Sci 2007;86:444-6. https://doi.org/ $10.1093 / \mathrm{ps} / 86.3 .444$

16.Zhang JL, Xie QM, Ji J, et al. Different combinations of probiotics improve the production performance, egg quality, and immune response of layer hens. Poult Sci 2012;91:2755-60. https://doi.org/10.3382/ps.2012-02339

17. Watkins BA, Kratzer FH. Effect of oral dosing of Lactobacillus strains on gut colonization and liver biotin in broiler chicks. Poult Sci 1983;62:2088-94. https://doi.org/10.3382/ps.0622088

18. Maiolino R, Fioretti A, Menna LF, Meo C. Research on the efficiency of probiotics in diets for broiler chickens. Nutr Abstr Rev Ser B 1992;62:482.

19. Chichlowski M, Croom J, McBride BW, Havenstein GB, Koci 
MD. Metabolic and physiological impact of probiotics or direct-fed-microbials on poultry: a brief review of current knowledge. Int J Poult Sci 2007;6:694-704. http://dx.doi.org/ 10.3923/ijps.2007.694.704

20.Fuller R. Probiotics in man and animals. J Appl Bacteriol 1989; 66:365-78.

21.Dibner JJ, Richards JD. Antibiotic growth promoters in agriculture: history and mode of action. Poult Sci 2005;84:634-43. https://doi.org/10.1093/ps/84.4.634

22. Patterson JA, Burkholder KM. Application of prebiotics and probiotics in poultry production. Poult Sci 2003;82:627-31. https://doi.org/10.1093/ps/82.4.627

23. Mohan B, Kadirvel R, Natarajan A, Bhaskaran M. Effect of probiotic supplementation on growth, nitrogen utilisation and serum cholesterol in broilers. Br Poult Sci 1996;37:395401. https://doi.org/10.1080/00071669608417870

24.Lan PT, Binh le T, Benno Y. Impact of two probiotic Lactobacillus strains feeding on fecal lactobacilli and weight gains in chicken. J Gen Appl Microbiol 2003;49:29-36. https://doi. org/10.2323/jgam.49.29

25. Khan M, Raoult D, Richet H, Lepidi H, La Scola B. Growthpromoting effects of single-dose intragastrically administered probiotics in chickens. Br Poult Sci 2007;48:732-5. https://doi. org/10.1080/00071660701716222

26. Awad WA, Ghareeb K, Abdel-Raheem S, Bohm J. Effects of dietary inclusion of probiotic and synbiotic on growth performance, organ weights, and intestinal histomorphology of broiler chickens. Poult Sci 2009;88:49-56. https://doi.org/10. 3382/ps.2008-00244

27. Angelakis E, Raoult $\mathrm{D}$. The increase of Lactobacillus species in the gut flora of newborn broiler chicks and ducks is associated with weight gain. PLoS One 2010;5:e10463. https://doi.org/10. 1371/journal.pone.0010463

28. Reid G, Burton J. Use of Lactobacillus to prevent infection by pathogenic bacteria. Microbes Infect 2002;4:319-24. https:// doi.org/10.1016/S1286-4579(02)01544-7 